\title{
Investigation of the Roles of Cyclooxygenase-2 and Galectin-3 Expression in the Pathogenesis of Premenopausal Endometrial Polyps
}

\author{
Esin Kasap · Serap Karaarslan ${ }^{1}$ \\ Esra Bahar Gur · Mine Genc \\ Nur Sahin · Serkan Güclü \\ Departments of Obstetrics and Gynecology and \\ 'Pathology, Sifa University School of Medicine, \\ Izmir, Turkey
}

\author{
Received: January 13, 2016 \\ Revised: March 6, 2016 \\ Accepted: March 8, 2016 \\ Corresponding Author \\ Esin Kasap, MD \\ Department of Obstetrics and Gynecology, \\ Sifa University School of Medicine, \\ Fevzipasa Boulvard, №. 172/2, 35240, \\ Basmane/lizmir, Türkiye \\ Tel: $+90-232-4460880$ \\ Fax: $+90-232-4460770$ \\ E-mail: dresincelik@windowslive.com
}

\begin{abstract}
Background: The pathogenesis and etiology of endometrial polyps has not been elucidated. In this study, we aimed to examine the pathogenic mechanisms of endometrial polyp development using immunohistochemistry. We evaluated the expression of galectin-3 and cyclooxgenase-2 (COX-2) during the menstrual cycle in premenopausal women with endometrial polyps or normal endometrium. Methods: Thirty-one patients with endometrial polyps and 50 healthy control patients were included in this study. The levels of expression of COX-2 and galectin-3 were studied by immunohistochemistry. Results: The percentage of COX-2-positive cells and the intensity of COX-2 staining in the endometrium did not vary during the menstrual cycle either in the control group or in patients with endometrial polyps. However, expression of galectin-3 was significantly lower in endometrial polyps and during the proliferative phase of the endometrium compared with the secretory phase. Conclusions: Our data suggests that the pathogenesis of endometrial polyps does not involve expression of COX-2 or galectin-3.
\end{abstract}

Key Words: Endometrial polyps; Cyclooxgenase-2; Galectin-3; Immunohistochemistry
Focal endometrial projections containing endometrial glands and stroma are termed endometrial polyps. ${ }^{1}$ The reported prevalence of endometrial polyps in premenopausal women with abnormal uterine bleeding is $33 \%$, but only $10 \%$ in asymptomatic women. ${ }^{2}$ While endometrial polyps can occur in women of all ages, they are more common in women between the ages of 40 and $49 .{ }^{3}$ Because endometrial polyps have not been reported to occur before the onset of menstruation, estrogenic stimulation is thought to be associated with endometrial polyp growth. ${ }^{4}$ However, the pathogenesis and etiology of endometrial polyps has not been clearly determined. Endometrial polyps are believed to form due to hormonal factors, e.g., estrogen and progesterone, which mediate endometrial proliferation and differentiation via steroid receptors, ${ }^{5}$ however, the mechanisms involved in the development of endometrial polyps are still unclear.

Cyclooxygenase (COX) is a key enzyme involved in the conversion of arachidonic acid to prostaglandins and other eicosanoids. Two isoforms of COX have been identified: COX-1 and COX-2. COX-1 is constitutively expressed in many tissues, whereas COX-2 is induced by a variety of factors including cy- tokines, growth factors, and tumor promoters. COX-2, which is involved in inflammatory responses and production of prostaglandins mediating uterine contractions, has been shown to induce excessive formation of some pro-angiogenic factors when overexpressed in colon cancer cell lines in vitro. ${ }^{6}$ Furthermore, recent studies have shown the influence of COX-2 in neoplastic development. ${ }^{5}$ However, the relationship between COX-2 and endometrial polyps has not been well established.

Galectin- 3 is a $\beta$-galactoside-binding animal lectin that contains carbohydrate-recognition domains and is involved in a multitude of biological tasks, ${ }^{7}$ including embryogenesis, growth, cell adhesion, proliferation, differentiation, cell-cycle progression, apoptosis, mRNA splicing, and immune system regulation. Galectin-3 is also involved in tumorigenesis, angiogenesis, and tumor metastasis, and is expressed in various cells and tissues including activated macrophages, eosinophils, neutrophils, mast cells, gastrointestinal and respiratory tract epithelial cells, kidneys cells, and some sensory neurons. ${ }^{7,8}$ Interestingly, extracellular galectin-3 plays a role in inflammation, while intracellular galectin- 3 participates in cell growth and anti-apoptotic processes 
and modulates cell adhesion and migration. ${ }^{7}$

Polyps tend to occur when apoptosis is inhibited and there is unopposed growth. ${ }^{9}$ However, the mechanisms mediating these endometrial alterations are unknown. Previous studies have suggested that endometrial polyps are a result of endometrial inflammation, i.e., endometritis, since the vessel axis of polyps has been shown to develop during endometritis. ${ }^{10}$ This finding suggests that identification of markers of inflammation and tissue growth may help to elucidate the pathogenic mechanisms of endometrial polyps. Indeed, recent studies have shown that the levels of expression of COX-2 and galectin-3 are increased during the progression from hyperplasia to cancer in the endometrial tissue, suggesting that these proteins may play a role in tumor cell function. However, the association of COX-2 and galectin-3 expression with polyps has not yet been established.

Therefore, in this study, we analyzed the levels of expression of COX-2 and galectin-3 in endometrial polyps and normal endometrium using immunohistochemistry.

\section{MATERIALS AND METHODS}

\section{Patients}

We examined a total of 81 cases of endometrial tissues in patients who were treated in the Department of Obstetrics and Gynecology of our hospital. Tissues were sampled between 2012 and 2014. All procedures were approved by the İzmir Sifa University Human Ethics Committee and followed the principles of the Declaration of Helsinki.

All patients were premenopausal (mean age, 37 years; range, 27 to 52 years) and had a recent history of regular menstruation (cycle of 25-35 days). None of the women included in the study used nonsteroidal anti-inflammatory drugs, hormone replacement therapy, or any other estrogen-containing pills. Thirty-one of the 81 patients had endometrial polyps, including 10 who had undergone hysterectomy and 21 who had undergone polypectomy and endometrial curettage. None of the patients had identifiable leiomyoma or adenomyosis by ultrasonography and/or magnetic resonance imaging. The control group consisted of samples from a total of 50 additional patients with normal endometrium, and included 23 samples collected during the proliferative phase and 27 samples collected during the secretory phase. Control patients were recruited from patients with benign ovarian cysts or a uterine prolapse but no other pathology, and the endometrial samples in this group were collected during hysterectomy procedures. Endometrial samples were grouped according to menstrual cycle phases: proliferative (days 1-14 of the cycle) and secretory (days 15-28 of the cycle). The day of the menstrual cycle was established from the women's menstrual history and confirmed by endometrial dating using the criteria described by Noyes et al. ${ }^{11}$

\section{Immunohistochemistry}

All tissue samples were fixed in $10 \%$ formalin and sent to pathology for analysis. Routine hematoxylin and eosin staining was carried out in all samples either to confirm the diagnosis of polyps or to date the endometrium. Samples were embedded in paraffin blocks, cut into 4 - $\mu$ m-thick sections, and deparaffinized. The sections were then stained with primary monoclonal antibodies against COX-2 (1:100, clone CX-294, Dako, Glostrup, Denmark) and galectin-3 (1:100, NCL-GAL3, clone 9C4, NovaCastra, Hamburg, Germany) using a Dako Cytomation Autostainer (Dako). After staining, each sample was evaluated under a light microscope (200×, Olympus BX53, Olympus, Tokyo, Japan) to determine the percentage of COX-2-positive cells, the intensity of COX-2 staining, and the percentage of galectin-3-positive cells. For positive controls, staining of breast carcinoma tissue for COX-2 and papillary thyroid carcinoma tissue for galectin-3 were used. Primary monoclonal antibodies were omitted in negative controls.

\section{Assessment of COX-2 and galectin-3 staining}

Semi-quantitative analysis of immunostaining for COX-2 and galectin-3 was performed as follows based on the percentage of cells with positive cytoplasmic staining: $0 \%, 0 ;<10 \%, 1 ; 10 \%-$ $50 \%, 2 ; 51 \%-80 \%, 3$; and $\geq 80 \%, 4$. In addition, staining intensity was evaluated as either negative $(0)$, weak $(1+)$, moderate $(2+)$, or strong $(3+)$. Semi-quantitative and intensity scores were analyzed separately. Additionally, the positivity of cells was evaluated as positive or negative. ${ }^{12}$ COX-2 and galectin- 3 expression was evaluated in glandular epithelial cells and stromal cells. Assessment of staining results was performed by one observer in a blinded fashion.

\section{Statistical analysis}

Statistical analysis was performed using software (Rstudio software ver. 0.98.501 via $\mathrm{R}$ language, $\mathrm{R}$ Studio Inc., Boston, MA, USA). Data describing continuous variables are presented as the mean \pm standard deviation. The Kruskal-Wallis and Pearson chi-square exact tests were used to compare continuous and categorical variables, respectively. Differences with p-values less than .05 were accepted as significant. 


\section{RESULTS}

\section{Patient demographics}

There was no statistically significant difference between the ages of healthy control individuals and patients with endometrial polyps.

\section{COX-2 expression}

Immunoreactivity for COX-2 was observed in glandular epithelial cells and stromal cells. COX-2-positive cells were predominantly observed in the endometrial glandular epithelium, where expression peaked during the secretory phase. COX2-positive cells were also observed in stromal cells, albeit to a lesser extent (Table 1, Fig. 1A). The percentage of COX-2-positive cells and the intensity of COX-2 staining in stromal cells and glandular epithelial cells did not vary during different periods of the menstrual cycle in the control group or in patients with endometrial polyps (Fig. 1B). Mean COX-2 scores in glandular epithelial cells and stromal cells were not significantly different between endometrial polyp specimens and normal endometrium specimens (Table 1).

\section{Galectin-3 expression}

Galectin-3 immunoreactivity was present in the endometrial glandular epithelial cells and stromal cells. Immunostaining was typically cytoplasmic. Galectin-3-positive cells were predominantly observed in the endometrial glandular epithelium, where expression levels peaked during the secretory phase. Galectin-3 expression was also observed to a lesser extent in stromal cells (Table 2). The mean percentage score of galectin-3 expression were lower both in endometrial polyps and the proliferative phase in normal endometrium than in the secretory phase in normal endometrium (Table 2, Fig. 1C). In glandular epithelial cells, no statistically significant differences in galectin-3 expression were found between endometrial polyps and normal endometrium during the proliferative phase. However, in patients with normal endometrium, galectin-3 expression was higher during the secretory phase $(\mathrm{p}=.349)$ (Fig. 1C). Finally, there

Table 1. Percentages of COX-2-positive cells and intensity of COX-2 staining

\begin{tabular}{|c|c|c|c|c|c|}
\hline & & Proliferative phase & Secretory phase & Endometrial polyps & $p$-value \\
\hline \multirow[t]{6}{*}{ Glandular epithelial tissues } & COX-2 intensity & $2.04 \pm 1.02$ & $2.07 \pm 0.83$ & $1.77 \pm 1.11$ & $.489^{a}$ \\
\hline & $0-1$ & 7 & 6 & 12 & $.398^{b}$ \\
\hline & $2-3$ & 16 & 21 & 19 & \\
\hline & COX-2 percentage score & $2.35 \pm 1.12$ & $2.67 \pm 1.04$ & $2.35 \pm 1.38$ & $.614^{\mathrm{a}}$ \\
\hline & $0-1$ & 4 & 3 & 8 & $.351^{\mathrm{b}}$ \\
\hline & $2-4$ & 19 & 24 & 23 & \\
\hline \multirow[t]{6}{*}{ Endometrial stroma } & COX-2 intensity & $0.65 \pm 0.71$ & $0.70 \pm 0.67$ & $0.53 \pm 0.68$ & $.564^{a}$ \\
\hline & $0-1$ & 20 & 24 & 27 & $.941^{b}$ \\
\hline & $2-3$ & 3 & 3 & 3 & \\
\hline & COX-2 percentage score & $0.61 \pm 0.65$ & $0.81 \pm 0.78$ & $0.61 \pm 0.76$ & $.525^{\mathrm{a}}$ \\
\hline & $0-1$ & 21 & 21 & 26 & $.430^{\mathrm{b}}$ \\
\hline & $2-4$ & 2 & 6 & 5 & \\
\hline
\end{tabular}

Values are presented as mean \pm standard deviation.

COX, cyclooxgenase.

${ }^{a} p$-value for Kruskal-Wallis tests, ${ }^{b} p$-value for Pearson chi-square test.
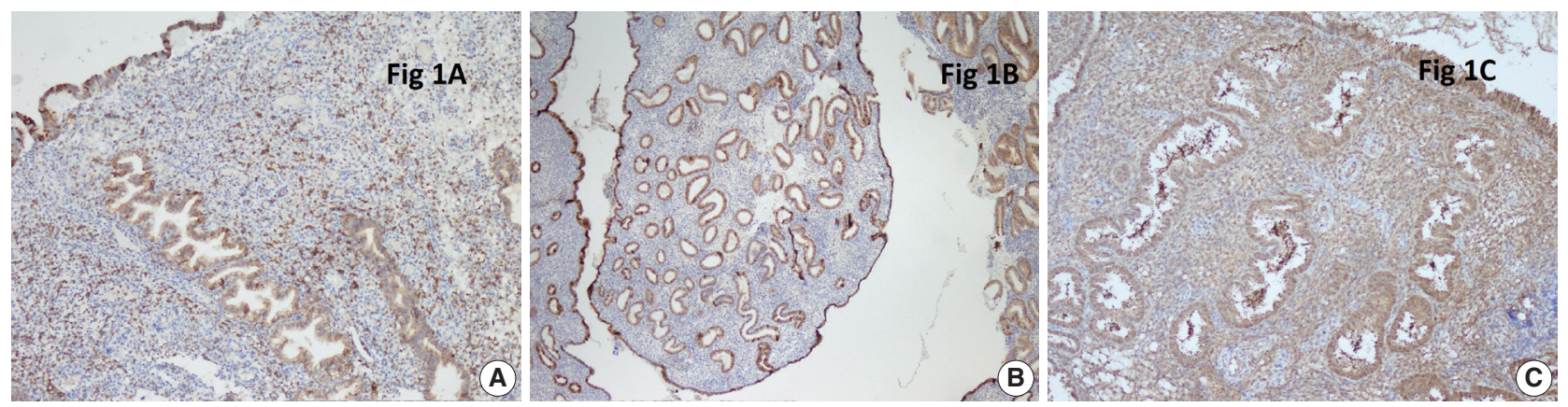

Fig. 1. Expression of cyclooxgenase 2 (COX-2) and galectin-3 in endometrium samples. (A) COX-2 expression in both the glandular epithelium and stroma during the secretory phase. (B) COX-2 expression in both the glandular epithelium and stroma of an endometrial polyp. (C) Galectin-3 expression in both the glandular epithelium and stroma during the secretory phase. 
Table 2. Percentages of galectin-3-positive cells

\begin{tabular}{lcccc}
\hline & Proliferative phase & Secretory phase & Endometrial polyp & $p$-value \\
\hline Galectin-3 glandular epithelial tissues (\% score) & $1.35 \pm 1.19$ & $2.19 \pm 1.00$ & $1.71 \pm 1.16$ & $.039^{\mathrm{a}}$ \\
Galectin-3 endometrial stroma (\% score) & $1.43 \pm 1.12$ & $1.63 \pm 1.15$ & $1.19 \pm 0.87$ & $.349^{\mathrm{a}}$ \\
\hline
\end{tabular}

Values are presented as mean \pm standard deviation.

${ }^{a} p$-value for Kruskal-Wallis tests.

were no differences in galectin- 3 expression in stromal cells of endometrial polyps and those of the endometrium at any phase of the menstrual cycle (Table 2).

\section{DISCUSSION}

In this study, we compared galectin- 3 and COX-2 expression and staining patterns using immunohistochemistry in endometrial polyps and normal endometrium during the secretory and proliferative phases. Our data indicated that both galectin- 3 and COX-2 were not associated with the formation of endometrial polyps.

Estrogen is known to play a pivotal role in the pathogenesis of endometrial malignant and benign cancers. However, the role of estrogen biosynthesis from stromal cells of the endometrium and its impact on malignancies has not been fully elucidated. Interestingly, estrogens are known to activate COX-2 in endometriosis, which increases levels of estradiol and prostaglandin above normal in women. ${ }^{13}$ Therefore, the pathogenesis of peritoneal endometriosis may be a consequence of enhanced prostaglandins in the eutopic endometrium that stimulate COX-2 expression and activity. ${ }^{14,15}$ This mechanism may also affect other endometrial pathologies, although no specific roles have been described.

COX-2 is expressed at higher levels in the glandular epithelial cells of the endometrium during the secretory phase of the menstrual cycle compared with the proliferative phase; however, its expression is similar during the secretory and proliferative phases in endometrial stromal cells. ${ }^{16}$ In the present study, we observed that COX-2 expression tended to be higher during the secretory phase than in either the proliferative phase or endometrial polyps in glandular epithelial cells.

Some studies have suggested that endometrial polyps may originate from endometrial inflammation. ${ }^{17}$ After demonstrating that COX-2 is expressed in the epithelial lining of postmenopausal polyps, Maia et al. ${ }^{18}$ concluded that COX-2 may be involved in the regulation of polyp growth. Nevertheless, COX-2 expression has not been shown to differ significantly between normal endometrial tissue and endometrial polyps. ${ }^{19}$

In 2006, Maia and colleagues compared COX-2 expression between endometrial polyps and normal endometrium in women with a regular menstrual cycle and found no significant difference in COX-2 expression between polyps and the normal endometrium. ${ }^{19}$ In addition, Pinheiro et al..$^{20}$ observed higher COX-2 expression in the glandular epithelium of obese women than in women of normal weight. This finding indicates that expression of COX-2 expression may be influenced by metabolic changes and growth factors associated with obesity. ${ }^{20}$ Here, we also demonstrated that COX-2 expression in the glandular epithelium and stromal cells in endometrial polyps did not differ significantly from that of normal endometrium. Thus, our data suggest that COX-2 expression may not be associated with the formation of endometrial polyps.

We previously reported that expression of galectin-3 is augmented during the formation of the receptive endometrium and the mid-secretory phase of the menstrual cycle. ${ }^{21}$ In addition, there is mounting evidence for a strong relationship between embryo implantation and tumor invasion and metastasis. Interestingly, galectin-3 has been reported to have anti-apoptotic potential in tumor cells, although there is no evidence for the role of galectin-3 in endometrial cell apoptosis. Thus, we hypothesize that galectin- 3 functions as an anti-apoptotic factor in the endometrium and may facilitate the development of endometrial polyps.

Chiu et al..$^{22}$ found that immunohistochemical analysis of galectin-3 protein expression is a sensitive, specific, and accurate marker for the diagnosis of thyroid cancer and certain other cancers. ${ }^{23}$ In endometriosis, Noel et al. ${ }^{24}$ found that galectin-3 protein expression, as measured using immunohistochemistry, is higher in endometriosis samples compared to eutopic endometrium, and also higher in the eutopic endometrium of women with endometriosis compared to those without endometriosis. Together, these data suggest that galectin-3 may have a potential role in the development of endometriosis. ${ }^{24}$ In a study by Brustmann et al. , $^{25}$ galectin-3 expression was assessed by immunohistochemistry in patients with normal endometrium, simple hyperplasia, complex hyperplasia without atypia, atypical hyperplasia, endometrioid carcinoma, serous papillary carcinoma, and clear cell carcinoma. They showed that in normal endometrium, simple hyperplasia, and complex hyperplasia without atyp- 
ia, the galectin-3 expression did not differ significantly among these groups. Immunohistochemical expression of galectin-3 for atypical hyperplasia and endometrioid carcinoma were significantly higher than those in the aforementioned groups. Serous papillary carcinoma and clear cell carcinoma, which are known to have a poorer prognosis than endometrioid carcinoma, ${ }^{26}$ also demonstrated significantly elevated galectin-3 expression levels in this study. On the basis of these findings, we assumed that galectin-3 expression may be related to the different biological behaviors of endometrialcarcinomas. ${ }^{25}$ Importantly, while there was no difference in galectin-3 expression in glandular epithelium and stromal cells of endometrial polyps and normal endometrium, our data demonstrated that galectin- 3 expression was significantly lower in both endometrial polyps and the proliferative phase of the endometrium than in the secretory phase of the endometrium. This finding suggests that larger-scale studies are needed to measure galectin- 3 levels in secretory adenocarcinomas, a subgroup of endometrioid adenocarcinomas, and in other endometrial cancers.

In analyzing our results, we wondered whether galectin-3 expression may be regulated by estrogen and progesterone. Although previous reports have shown that estrogen and/or progesterone increases galectin- 3 expression in endometrial cells at all concentrations, ${ }^{27}$ we did not observe any direct effect of these hormones on galectin- 3 expression, suggesting that the relationship may be indirect. Thus, further studies are needed to determine the exact nature of the relationship of estrogen and progesterone on galectin-3 expression.

The small sample size was a limitation of the present study. Therefore, the results of this study must be confirmed in larger longitudinal population studies.

In summary, our data suggested that endometrial polyps may form through certain common pathways that do not involve COX-2 and galectin-3. While COX-2 and galectin-3, in addition to angiogenesis, are known to be involved in different steps of carcinogenesis, ${ }^{28}$ these two target proteins may not be involved in the formation of endometrial polyps. Further large scale studies comparing adjacent endometrial tissue and healthy women at specific phases of the menstrual cycle should be performed.

\section{Conflicts of Interest}

No potential conflict of interest relevant to this article was reported.

\section{REFERENCES}

1. Neto LC, Soares JM Jr, Giusa-Chiferi MG, Gonçalves WJ, Baracat EC. Expression of p53 protein in the endometrial polyp in postmenopausal women. Eur J Gynaecol Oncol 2013; 34: 509-12.

2. Clevenger-Hoeft M, Syrop CH, Stovall DW, Van Voorhis BJ. Sonohysterography in premenopausal women with and without abnormal bleeding. Obstet Gynecol 1999; 94: 516-20.

3. Droegemueller W. Benign gynecologic lesions. In: Stenchever MA, Droegemueller W, Herbst AL, Mishell DR Jr, eds. Comprehensive gynecology. 5th ed. St. Louis: Mosby Inc., 2001; 440-92.

4. Inceboz US, Nese N, Uyar Y, et al. Hormone receptor expressions and proliferation markers in postmenopausal endometrial polyps. Gynecol Obstet Invest 2006; 61: 24-8.

5. Sant'Ana de Almeida EC, Nogueira AA, Candido dos Reis FJ, Zambelli Ramalho LN, Zucoloto S. Immunohistochemical expression of estrogen and progesterone receptors in endometrial polyps and adjacent endometrium in postmenopausal women. Maturitas 2004; 49: 229-33.

6. Kendall RL, Wang G, Thomas KA. Identification of a natural soluble form of the vascular endothelial growth factor receptor, FLT-1, and its heterodimerization with KDR. Biochem Biophys Res Commun 1996; 226: 324-8.

7. Lee J, Moon C, Kim J, et al. Immunohistochemical localization of galectin-3 in the granulomatous lesions of paratuberculosis-infected bovine intestine. J Vet Sci 2009; 10: 177-80.

8. Sakaki M, Fukumori T, Fukawa T, et al. Clinical significance of galectin-3 in clear cell renal cell carcinoma. J Med Invest 2010; 57: 152-7.

9. Topcu HO, Erkaya S, Guzel AI, et al. Risk factors for endometrial hyperplasia concomitant endometrial polyps in pre- and postmenopausal women. Asian Pac J Cancer Prev 2014; 15: 5423-5.

10. Costa-Paiva L, Godoy CE Jr, Antunes A Jr, Caseiro JD, Arthuso M, Pinto-Neto AM. Risk of malignancy in endometrial polyps in premenopausal and postmenopausal women according to clinicopathologic characteristics. Menopause 2011; 18: 1278-82.

11. Noyes RW, Hertig AT, Rock J. Dating the endometrial biopsy. Fertil Steril 1950; 1: 3-25.

12. Harrington DJ, Lessey BA, Rai V, et al. Tenascin is differentially expressed in endometrium and endometriosis. J Pathol 1999; 187: 242-8.

13. Maia H Jr, Maltez A, Studard E, Zausner B, Athayde C, Coutinho E. Effect of the menstrual cycle and oral contraceptives on cyclooxygenase-2 expression in the endometrium. Gynecol Endocrinol 2005; 21: 57-61.

14. Erdemoglu E, Güney M, Karahan N, Mungan T. Expression of cyclooxygenase-2, matrix metalloproteinase-2 and matrix metallo- 
proteinase-9 in premenopausal and postmenopausal endometrial polyps. Maturitas 2008; 59: 268-74.

15. Bulun SE, Zeitoun KM, Takayama K, Sasano H. Estrogen biosynthesis in endometriosis: molecular basis and clinical relevance. J Mol Endocrinol 2000; 25: 35-42.

16. Wang D, Chen Q, Zhang C, Ren F, Li T. DNA hypomethylation of the COX-2 gene promoter is associated with up-regulation of its mRNA expression in eutopic endometrium of endometriosis. Eur J Med Res 2012; 17: 12.

17. Carvalho FM, Aguiar FN, Tomioka R, de Oliveira RM, Frantz N, Ueno J. Functional endometrial polyps in infertile asymptomatic patients: a possible evolution of vascular changes secondary to endometritis. Eur J Obstet Gynecol Reprod Biol 2013; 170: 152-6.

18. Maia H Jr, Correia T, Freitas LA, Athayde C, Coutinho E. Cyclooxygenase-2 expression in endometrial polyps during menopause. Gynecol Endocrinol 2005; 21: 336-9.

19. Maia H Jr, Pimentel K, Silva TM, et al. Aromatase and cyclooxygenase-2 expression in endometrial polyps during the menstrual cycle. Gynecol Endocrinol 2006; 22: 219-24.

20. Pinheiro A, Antunes A Jr, Andrade L, De Brot L, Pinto-Neto AM, Costa-Paiva L. Expression of hormone receptors, Bcl2, Cox2 and Ki67 in benign endometrial polyps and their association with obesity. Mol Med Rep 2014; 9: 2335-41.
21. Du GP, Zhang W, Wang L, Liu YK, Zhou JP. Expression of galectin-3 in human endometrium. Fudan Univ J Med Sci 2006; 2: 143-6.

22. Chiu CG, Strugnell SS, Griffith OL, et al. Diagnostic utility of galectin-3 in thyroid cancer. Am J Pathol 2010; 176: 2067-81.

23. Cay T. Immunhistochemical expression of galectin-3 in cancer: a review of the literature. Turk Patoloji Derg 2012; 28: 1-10.

24. Nöel JC, Chapron C, Borghese B, Fayt I, Anaf V. Galectin-3 is overexpressed in various forms of endometriosis. Appl Immunohistochem Mol Morphol 2011; 19: 253-7.

25. Brustmann H, Riss D, Naudé S. Galectin-3 expression in normal, hyperplastic, and neoplastic endometrial tissues. Pathol Res Pract 2003; 199: 151-8.

26. Silverberg SG, Kurman RJ. Atlas of tumor pathology, 3rd series. Fascicle 3. Tumors of the uterine corpus and gestational trophoblastic disease. Washington, DC: Armed Forces Institute of Pathology, 1992.

27. Yang H, Lei C, Cheng C, et al. The antiapoptotic effect of galectin-3 in human endometrial cells under the regulation of estrogen and progesterone. Biol Reprod 2012; 87: 39.

28. Kumar V, Abbas AK, Fausto N. Robbins and Cotran: pathologic basis of disease. 7th ed. Philadelphia: Elsevier Saunders, 2005; 269342. 